\title{
As aves da Estação Ecológica Serra Geral do Tocantins, Centro do Brasil
}

\author{
Marco Antonio Rego ${ }^{1}$, Luís Fábio Silveira ${ }^{1,3}$, Vítor de Queiroz Piacentini, Fabio Schunck, \\ Érika Machado ${ }^{1}$, Renato Torres Pinheiro ${ }^{2}$ \& Elivânia Reis ${ }^{2}$ \\ ${ }^{1}$ Departamento de Zoologia, Museu de Zoologia, Universidade de São Paulo - USP, \\ CP 114161, CEP 05422-970, São Paulo, SP, Brasil \\ ${ }^{2}$ Grupo de Pesquisa em Ecologia e Conservação de Aves, Universidade Federal do Tocantins - UFT, \\ CP 114, CEP 77001-090, Palmas, TO, Brasil \\ ${ }^{3}$ Autor para correspondência: Luís Fábio Silveira, e-mail: lfsilvei@usp.br
}

REGO, M.A., SILVEIRA, L.F., PIACENTINI, V.Q., SCHUNCK, F., MACHADO, E., PINHEIRO, R.T. \& REIS, E. The birds of Estação Ecológica Serra Geral do Tocantins. Biota Neotrop. 11(1): http://www.biotaneotropica. org.br/v11n1/en/abstract?article+bn03711012011.

\begin{abstract}
The Estação Ecológica Serra Geral do Tocantins is one of the largest conservation units in Cerrado Biome. The avifauna of this locality was studied from January 25 to 15 February, 2008. We attempted to sample the different habitats found both within the EESGT as in its surroundings. In addition to audio and visual records, mist nets and shotguns were used to collect testimony material, which was deposited in the ornithological collection of the Museu de Zoologia da Universidade de São Paulo (MZUSP). The results were compared with data available from other conservation units nearby the EESGT. We recorded 254 bird species; among these 11 were considered endemic to the Cerrado region and other three considered threatened (Taoniscus nanus, Anodorhynchus hyacinthinus and Procnias averano). Three individuals belonging to the Picumnus genus also were collected; they presented unusual plumage and vocalization patterns, which suggests an undescribed taxon. Keywords: Estação Ecológica Serra Geral do Tocantins, Cerrado, avifauna, inventory.
\end{abstract}

REGO, M.A., SILVEIRA, L.F., PIACENTINI, V.Q., SCHUNCK, F., MACHADO, E., PINHEIRO, R.T. \& REIS, E. As aves da Estação Ecológica Serra Geral do Tocantins. Biota Neotrop. 11(1): http://www.biotaneotropica. org.br/v11n1/pt/abstract?article+bn03711012011.

Resumo: A Estação Ecológica Serra Geral do Tocantins (EESGT) é uma das maiores Unidades de Conservação dentro do Bioma Cerrado. A avifauna desta UC foi inventariada entre os dias 25 de janeiro e 15 de fevereiro de 2008. Durante esse trabalho buscou-se amostrar os diferentes hábitats encontrados na EESGT, bem como algumas localidades no entorno. Além de registros auditivos e visuais foram utilizadas redes-de-neblina e armas de fogo para coletas de material testemunho, que se encontra depositado na coleção ornitológica do Museu de Zoologia da Universidade de São Paulo (MZUSP). Os resultados obtidos foram comparados com os dados disponíveis para outras unidades de conservação presentes na região. Foram registradas 254 espécies de aves, incluindo 11 endêmicas do Cerrado e outras três ameaçadas de extinção (Taoniscus nanus, Anodorhynchus hyacinthinus e Procnias averano). Três indivíduos pertencentes ao gênero Picumnus foram coletados; estes apresentam um distinto padrão de plumagem e de vocalização, podendo representar um táxon ainda não descrito.

Palavras-chave: Estação Ecológica Serra Geral do Tocantins, Cerrado, avifauna, inventário. 


\section{Introdução}

O Cerrado é o segundo maior bioma da América do Sul, ocupando toda a região do Brasil central, além do sudeste boliviano e a região nordeste do Paraguai (Ab’Saber 1977, Ratter et al. 1997). Embora mais de 800 espécies de aves já tenham sido registradas neste bioma (Silva 1995b, Silva \& Santos 2005), sua diversidade ainda não é completamente conhecida, com espécies novas sendo descritas nas proximidades de grandes centros urbanos (Zimmer et al. 2001) e muitas áreas do Cerrado ainda são consideradas como insuficientemente amostradas mesmo depois da compilação realizada por Silva (1995a).

A região da Serra Geral, situada na divisa entre os Estados da Bahia com Tocantins e Goiás, é um bom exemplo de área dentro deste bioma ainda não suficientemente amostrada. A Serra Geral, ao norte, se encontra com a Chapada das Mangabeiras, local onde nasce o Rio Parnaíba e alguns de seus principais afluentes, próximo a divisa entre os Estados do Tocantins, Piauí e Maranhão, estendendo-se para o sul até próximo a Serra do Ramalho, no sudoeste da Bahia (Instituto... 2006).

A avifauna desta região só começou a ser conhecida a partir da década de 1910, quando o alemão Rudolph Pfrimer colecionou algumas aves, posteriormente enviadas para o Museu Nacional, no Rio de Janeiro (Pacheco 2004). Entretanto, este material não apresenta informações confiáveis em relação a data e sexo desses exemplares, sendo que muitos espécimes se misturaram a outros provenientes da Comissão Rondon, oriundos do Mato Grosso, também presentes na mesma instituição. Uma possível explicação para esta mistura de material pode ser creditada à substituição, já no Museu Nacional, das etiquetas originais de Pfrimer por outras confeccionadas por Alípio de Miranda Ribeiro (Pacheco 2004, Pacheco \& Olmos 2006).

Outros coletores ou naturalistas também tangenciaram a região da Serra Geral, destacando-se Otmar Reiser, R. H. Becker e Emil Kaempfer. Reiser, em 1903, integrou a equipe liderada pelo ictiólogo Franz Steindachner na primeira expedição enviada para amostrar as nascentes do Rio Parnaíba, no Sul do Piauí. Reiser descreveu espécies de aves interessantes como o bico-virado-dacaatinga, Megaxenops parnaguae, além de observar a ararinha-azul, Cyanopsitta spixii, nos arredores de Parnaguá (Naumburg 1928, Hellmayr 1929). Becker amostrou o noroeste baiano, na região do município de São Marcelo (próximo ao limite leste da EESGT) entre março e abril de 1913. Nesta região, onde predominam ambientes típicos de Cerrado e de transição deste com a Caatinga, foram coletados táxons característicos dos dois biomas e alguns endêmicos do Cerrado como Alipiopsitta xanthops e Saltatriculla atricollis (Cory 1918, Hellmayr 1938, Pacheco 2004).

Posteriormente, Emil Kaempfer, contratado pelo American Museum of Natural History, atravessou todo o leste do país, desde o Maranhão até Rio Grande do Sul e, em meados de 1927, passou pelas áreas amostradas por Reiser (Naumburg 1928, 1935, Paynter \& Traylor 1991). Desde então, toda a região da Serra Geral foi negligenciada nas amostragens da avifauna do Cerrado, e apenas recentemente, diversos autores (Santos 2001, Pacheco \& Silva 2002, Tocantins 2003, Braz 2003, Braz et al. 2003, Leite \& Lopes 2002, Lopes 2007, 2008) trabalharam na região da Serra das Mangabeiras, Jalapão e Serra Geral. A criação das Unidades de Conservação (UC) do Parque Estadual do Jalapão (PEJ), das Áreas de Proteção Ambiental da Serra de Tabatinga e da Chapada das Mangabeiras (APATM) e do Parque Nacional das Nascentes do Parnaíba também podem ser apontadas como fatores importantes para o incremento das pesquisas na região.

Fazendo parte deste mosaico de Unidades de Conservação, destaca-se a Estação Ecológica Serra Geral do Tocantins (EESGT). Desde a sua criação, em 2001, nenhum inventário avifaunístico havia sido realizado nesta UC. Com o objetivo de se conhecer a avifauna desta Estação Ecológica, foram realizadas amostragens, entre 25 de janeiro e 15 de fevereiro de 2008, em diferentes ambientes dentro da EESGT e nos seus arredores. No presente estudo são apresentados os resultados obtidos nesta campanha, sendo os mesmos comparados com os dados disponíveis para amostragens prévias na região, em estudos no Parque Estadual do Jalapão e as Áreas de Proteção Ambiental da Serra de Tabatinga e da Chapada das Mangabeiras.

\section{Metodologia}

A EESGT possui 716.306 ha e se situa entre a porção leste do Estado do Tocantins, nos municípios de Almas, Mateiros, Ponte Alta e Rio da Conceição, e no oeste baiano, no município de Formosa do Rio Preto, entre as coordenadas $10^{\circ} 30^{\prime}-11^{\circ} 17^{\prime} \mathrm{S}$ e $47^{\circ} 14^{\prime}-46^{\circ} 10^{\prime} \mathrm{W}$ (Brasil 2001). A EESGT é recoberta principalmente por vegetações típicas de Cerrado, predominando formações mais campestres como campo sujo e campo cerrado, afloramentos calcários, e cerrado sensu stricto. As grandes porções de áreas abertas são cortadas por florestas ciliares, veredas e áreas alagadas. A EESGT está inserida em parte das bacias hidrográficas dos Rios Tocantins e São Francisco e a presença de grandes chapadões em contato com depressões e escarpas de arenito constitui uma paisagem complexa e única. Essa região apresenta uma das menores densidades demográficas do Brasil e em boa parte de sua área a ocupação humana é mínima (Mamede et al. 2002, Tocantins 2003).

Com o intuito de suprir as deficiências particulares dos métodos de amostragens de aves, optou-se por vários métodos simultâneos, que também tornaram o trabalho de campo mais produtivo, gerando resultados de alta qualidade e confiabilidade em um curto espaço de tempo. Registros visuais e auditivos foram feitos durante todo o trajeto dentro de áreas selecionadas, enquanto que os demais métodos de coleta foram concentrados nas áreas previamente selecionadas. As espécies foram identificadas visualmente com o auxílio de binóculos $10 \times 40$ e $8,5 \times 45$. As diversas manifestações sonoras emitidas pelas aves foram gravadas em cassete e em meio digital (gravadores Sony TCM 5000EV, Sony Hi-MD MZM 100 e Sony MZ-RH1 e microfone Sennheiser ME 66). Para capturar as aves foram utilizadas redes-de-neblina, estendidas em transeções abertas em cada uma das áreas escolhidas. Para amostrar algumas espécies de aves que habitam estratos mais elevados da vegetação, mais difíceis de serem capturadas por redes-de-neblina, ou que, por seu comportamento e/ou tamanho não são amostradas por este métodos, foram utilizadas espingardas calibres $20,28,36$ e .22. Todas as aves coletadas foram taxidermizadas.

Dados biométricos (massa e comprimento total) e coloração das partes nuas foram anotados, além da eventual coleta de ectoparasitos. Amostras de tecido (musculatura peitoral) foram retiradas de todas as aves coletadas. As carcaças foram mantidas em álcool $70 \%$ v.v. Todo o material foi depositado no Museu de Zoologia da Universidade de São Paulo.

Os trabalhos de campo iniciaram-se antes do nascer do sol e se estenderam até por volta das 20 horas, para a observação de aves noturnas. As redes-de-neblina foram abertas ao nascer do sol e fechadas por volta das onze horas da manhã. As redes-de-neblina (19 em cada área, $12 \mathrm{~m}$ de comprimento e 2,40 m de altura - malha de $36 \mathrm{~mm}$ ) foram montadas em linha, permanecendo por quatro dias em cada localidade selecionada, totalizando 1.520 horas-rede. Em cada uma das localidades selecionadas foram estendidas duas linhas de rede, uma em cada microhábitat, cobrindo $228 \mathrm{~m}$ em cada um dos pontos, sendo as linhas abertas simultaneamente.

Entre 26 e 30 de janeiro amostramos a porção sul da EESGT, no município de Rio da Conceição (10³9' S e 46 48' W). Nesta 
área metade das redes foi instalada em uma vereda, enquanto que as demais foram montadas em uma porção de Cerrado sensu stricto adjacente. As redes foram novamente abertas entre 8 e 12 de fevereiro, a nordeste da EESGT, no município de Mateiros, próximo à mata do Rio Galhão $\left(10^{\circ} 31^{\prime} \mathrm{S}\right.$ e $\left.46^{\circ} 06^{\prime} \mathrm{W}\right)$, em hábitats semelhantes ao do primeiro ponto. Em outras localidades, como o Vale Encantado ( $11^{\circ} 37^{\prime} \mathrm{S}$ e $46^{\circ} 42^{\prime} \mathrm{W}$, afloramentos calcários e mata seca), a Mata do Rio Galhão, $\left(10^{\circ} 31^{\prime} \mathrm{S}\right.$ e $\left.46^{\circ} 06^{\prime} \mathrm{W}\right)$ e a estrada da soja (10 $56^{\circ} \mathrm{S}$ e $46^{\circ} 18^{\prime} \mathrm{W}$ ) não foram abertas redes-de-neblina, sendo utilizados os demais métodos para o inventário. Nestas áreas os trabalhos duraram, em média, um dia.

\section{Resultados e Discussão}

Em 17 dias de campo foram registradas 254 espécies (Apêndice 1) pertencentes a 54 famílias, sendo 125 ( $50 \%)$ delas representadas por material testemunho (peles em via seca). As famílias com o maior número de representantes foram Tyrannidae (41), Emberizidae (17), Psittacidae (13) e Accipitridae (13). Onze das 254 espécies são consideradas como endêmicas do Cerrado, segundo Silva (1995b, 1997) e Silva \& Santos (2005), representando cerca de $37 \%$ dos endemismos desse Bioma (Tabela 1). Outras espécies características de formações de Cerrado também foram muito comuns na EESGT, como Heliactin bilophus, Neothraupis fasciata e Cypsnagra hirundinacea.

De acordo com Silva (1995b) e Silva \& Santos (2005), cerca de $70 \%$ das aves que ocorrem no Cerrado são dependentes ou semi-dependentes das formações florestais existentes neste Bioma, enquanto que 218 espécies não dependem das florestas para se alimentar e/ou se reproduzir. Foram registradas 118 espécies consideradas como independentes das formações florestais, o que corresponde a 54\% das espécies não dependentes de florestas listadas por Silva \& Santos (2005) para todo o bioma. Este número demonstra a importância da EESGT para a conservação das espécies típicas de Cerrado, uma vez que uma proporção significativa das espécies registradas depende inteiramente desta formação vegetal.

Três táxons ameaçados de extinção e seis quase-ameaçados (near threatened - NT) foram registrados (Machado et al. 2008, International... 2009). As espécies consideradas como quase-ameaçadas são: Rhea americana, Alipiopsitta xanthops, Euscarthmus rufomarginatus, Knipolegus franciscanus, Neothraupis fasciata e Charithospiza eucosma (International... 2009). Entre as espécies ameaçadas estão Taoniscus nanus, que se enquadra na categoria de vulnerável (VU), Anodorhynchus hyacinthinus, considerada em perigo (EN), e Procnias averano, considerada em perigo apenas pelo MMA (Machado et al. 2008).

De maneira geral a avifauna da EESGT encontra-se bem conservada. Nas veredas, espécies como Ara ararauna (MZUSP 79505 e 79506), Berlepschia rikeri (e.g. MZUSP 79660), Tyrannopsis sulphurea (MZUSP 79730 e 79731) e Basileuterus leucophrys (e.g. MZUSP 79746) eram bastante abundantes. Nas áreas pedregosas, onde eram observadas diferentes espécies de canelas-de-ema (Vellozia sp.), aves como Sicalis citrina e Buteo melanoleucus (MZUSP 79481 e 79482) foram registradas. Entretanto, aves características desta formação, como Porphyrospiza caerulescens, registrada tanto no PEJ (Pacheco \& Silva 2002) quanto no APATM (Santos 2001), não foram vistas na EESGT, o que pode ser apenas um viés na amostragem. Fora da EESGT, no Vale Encantado, foi observada uma formação florestal associada com afloramentos de calcário, onde eram comuns as barrigudas (Ceiba sp.), árvores típicas das matas secas que também são observadas no norte de Minas Gerais e no sudoeste baiano. Nesta região espécies típicas dos afloramentos calcários como Knipolegus franciscanus
Tabela 1. Espécies endêmicas do Cerrado registradas na EESGT e adjacências no presente trabalho

Table 1. Cerrado endemics birds recorded at EESGT and surrounding areas.

\begin{tabular}{ll}
\hline \multicolumn{1}{c}{ Nome do táxon } & \multicolumn{1}{c}{ Nome em português } \\
\hline Taoniscus nanus & Inhambu-carapé \\
Alipiopsitta xanthops & Papagaio-galego \\
Melanopareia torquata & Tapaculo-de-colarinho \\
Herpsilochmus longirostris & Chorozinho-de-bico-comprido \\
Suiriri islerorum & Suiriri-da-chapada \\
Knipolegus franciscanus & Maria-preta-do-nordeste \\
Antilophia galeata & Soldadinho \\
Cyanocorax cristatellus & Gralha-do-campo \\
Charitospiza eucosma & Mineirinho \\
Saltatricula atricollis & Bico-de-pimenta \\
Basileuterus leucophrys & Pula-pula-de-sobrancelha \\
\hline
\end{tabular}

(MZUSP 79681 e 79682), foram facilmente encontradas, o que reforça a associação desta espécie com este tipo particular de floresta seca (Lima 1999). Nesta formação florestal foram realizados os únicos registros de Herpsilochmus atricapillus (MZUSP 79638) e Picumnus albosquamatus (MZUSP 79614), que são substituído na EESGT, respectivamente, por $H$. longirostris (e.g. MZUSP 79636 e 79637) e Picumnus sp. (MZUSP 79615-79617). Outras espécies registradas apenas neste local foram Momotus momota, Nonnula rubecula (MZUSP 79594) e Patagioenas plumbea, espécies mais associadas à ambientes florestais. Registros relevantes e informações biológicas para algumas espécies da EESGT e arredores são destacados abaixo.

Rhea americana: relativamente rara na EESGT, foi bastante comum fora da UC, na região de Mateiros, onde adultos com grupos de até 18 filhotes foram observados. Sua raridade na EESGT pode ser devido às queimadas que aconteceram nos últimos anos. Fora da EESGT esta espécie foi encontrada sempre próxima a plantações, como observado também por Pinheiro \& Dornas (2009) na Área de Proteção Ambiental da Ilha do Bananal e no Parque Estadual do Cantão.

Taoniscus nanus: menor espécie da família, cuja detecção é bastante difícil. Prefere áreas de campo limpo, seguida por aquelas de campo sujo (Silveira \& Silveira 1998). Sua identificação em campo depende muito do reconhecimento de sua voz, semelhante à emitida por grilos (Orthoptera)(Sick 1997, Silveira \& Silveira, 1998, Silveira 2008a). Três indivíduos desta espécie foram observados (mas nenhum ouvido) em três ocasiões e localidades distintas dentro da EESGT, todas elas na sua porção norte. É naturalmente raro e costuma ocorrer nas mesmas áreas com a igualmente ameaçada Nothura minor (Silveira \& Silveira 1998, Silveira 2008b), que não foi registrada na EESGT. A presença de Taoniscus nanus na EESGT está diretamente ligada ao controle das queimadas na região, pois esta espécie tem limitada capacidade de vôo e de dispersão.

Chondrohierax uncinatus: amplamente distribuída na região neotropical (Pinto 1964, Sick 1997), apresenta hábitos gregários e migratórios ainda pouco conhecidos. Entre os dias 9 e 12 de fevereiro foi possível observar dezenas de indivíduos voando em conjunto sobre a EESGT e o Vale Encantado. Os indivíduos observados apresentaram a plumagem variando desde negro e cinza até aqueles com ventre branco-puro, passando pelas diferentes formas com barras no ventre. Uma fêmea foi coletada (MZUSP 79860); esta apresentava a plumagem barrada de marrom, uma das formas mais características 
desta espécie altamente polimórfica. Não foram ouvidas vocalizações e nenhum outro indivíduo foi observado na região depois do dia 12/2.

Anodorhynchus hyacinthinus: a arara-azul-grande foi observada em quatro diferentes ocasiões e localidades, sempre em grupos reduzidos ou pares. Aparentemente não há pressão para captura de filhotes ou adultos para o comércio ilegal de aves silvestres, e a espécie nidifica, na região, em paredões de arenito, especialmente na área conhecida como Pedra do Baú. As escarpas de arenito presentes no Morro do Fumo, Bigorna e em grande parte da escarpa da Serra Geral, dentro da EESGT, são importantes sítios reprodutivos desta espécie (C. Nogueira, com. pess.). A EESGT situa-se a menos de $100 \mathrm{~km}$ a oeste de Formosa do Rio Preto onde, há cerca de 35 anos, um registro de Cyanopsitta spixii foi reportado (Collar et al. 1992, Sick 1997, Santos 2001). Entretanto, nem mesmo a população mais tradicional reconhece a presença desta espécie na área.

Asio flammeus: coruja típica de áreas abertas, frequentemente vista caçando próximo ao solo (Sick 1997). Registros para o Cerrado eram raros até meados da década de 1990 (Silveira 1998, Bagno \& Rodrigues 1998, Silva \& Santos 2005, Lopes et al. 2008). Espécie relativamente comum nas extensas plantações de soja adjacentes à EESGT, onde podia ser vista mesmo durante o dia. A presença de Asio flammeus em plantações de soja é também observada no entorno do Parque Nacional das Emas, onde esta coruja é muito mais comum nesta monocultura do que dentro do parque (Silveira, obs. pess.), mesma situação observada na EESGT. Uma possível explicação para este fato pode estar relacionado a uma maior abundância de pequenos roedores nas plantações de soja do que nas formações naturais. Entretanto, esta hipótese ainda carece de estudos mais conclusivos.

Colaptes campestris: duas formas são atualmente aceitas como componentes deste táxon, a forma nominal e $C$. c . campestroides (Winkler \& Christie 2002), que se diferenciam pela cor da garganta (negra na forma nominal, branca em C. c. campestroides), embora padrões intermediários já tenham sido descritos (Short 1982). Entretanto, outras raças geográficas já foram descritas com base nos padrões de estrias da região ventral, que pode se apresentar na forma de setas ou estrias (Short 1982). Em duas áreas opostas dentro da EESGT foram coletados indivíduos (MZUSP 79611 e 79612) que apresentam plumagens muito distintas na sua região ventral, sugerindo um polimorfismo sem valor taxonômico, L. Novaes, em prep.

Picumnus sp.: diversos indivíduos de um pica-pau-anão foram observados e ouvidos em diversas áreas da EESGT e entorno. A vocalização é distinta tanto de P. albosquamatus, que só foi registrado nas matas secas da região do Vale Encantado (MZUSP 79614) quanto de $P$. pygmaeus, outra espécie que potencialmente poderia ocorrer na região, sendo registrada por Santos (2001) na APATM, mas não no presente estudo. Três indivíduos foram coletados em localidades distintas, e todos apresentam um padrão de plumagem semelhante entre si e distinto de qualquer outra espécie de Picumnus. A vocalização foi gravada e é também distinta de qualquer outra espécie do gênero. Estes pica-paus só foram registrados em áreas de mata ciliar, sempre em baixa densidade. Conclusões sobre a presença de uma espécie ainda não descrita são preliminares, pois a hibridação entre os representantes deste gênero é um fenômeno bem conhecido (Short 1982, Lencioni Neto 1995). Entretanto, a congruência do padrão de plumagem entre os três indivíduos de localidades distintas e a ausência de registros de uma segunda espécie do gênero sugere que pode se tratar de um táxon ainda não descrito e que se encontra em estudo.

Suiriri islerorum: espécie descrita recentemente (Zimmer et al. 2001), pode ser facilmente separada em campo da sua congênere Suiriri s. affinis através da vocalização e comportamento (Lopes 2005; Lopes \& Marini 2005). Ocorrem em sintopia com S. s. affinis na EESGT, sendo freqüentes em áreas de Cerrado sensu strictu.
Os exemplares coletados (MZUSP 79707-79715) apresentavam as gônadas pouco desenvolvidas (Lopes \& Marini 2005).

Procnias averano: espécie com distribuição disjunta, sendo consideradas duas populações distintas, uma presente na Venezuela, na ilha de Trinidad, e em Roraima e, outra que se distribui pela Floresta Atlântica do Nordeste e Matas Secas do interior (Snow 1982, Ridgely \& Tudor 1994). Foram registrados diversos machos cantando nas matas ciliares e veredas, onde a espécie é bem conhecida da população como ave migratória. Em uma área de aproximadamente dois quilômetros foram ouvidos pelo menos seis machos.

Basileuterus leucophrys: Endemismo típico das matas ciliares e amplamente distribuído pelo Cerrado (Silva 1997), ocorrendo marginalmente no Pantanal. Encontrada quase sempre aos pares e sempre associado à água, é comum no Parque Nacional de Brasília (Ridgely \& Tudor 1989). Na EESGT também foi encontrada em abundância, sendo registrada em três dos quatro dias que permanecemos na localidade de Rio da Conceição, e encontrada também nas veredas, especialmente nas áreas de matas alagadas. Os registros apresentados neste trabalho se aproximam do limite norte da distribuição dessa espécie, encontrada também no PEJ (Tocantins 2003)

O presente estudo registrou 33 espécies não registradas nos trabalhos anteriores, dentre elas algumas associadas a ambientes amazônicos (Stotz et al. 2006), como Celeus flavus e Tyrannopsis sulphurea, e outras que são naturalmente incomuns, e.g. Spizaetus melanoleucus. A presença de Suiriri islerorum apenas na EESGT provavelmente se deve ao fato de que até a sua descrição por Zimmer et al. (2001) essa espécie era confundida com S. s. affinis.

Somando-se as listas disponíveis para o PEJ (Pacheco \& Silva 2002, Leite \& Lopes 2002, Braz 2003, Tocantins 2003), para a APATM (Santos 2001) e a do presente trabalho, chega-se a um total de 366 espécies para a região da Serra Geral e áreas adjacentes, na região do Jalapão, nas fronteiras dos Estados do Tocantins, Maranhão, Bahia e Piauí (Apêndice), o que corresponde a 42\% da avifauna já registrada no Bioma Cerrado segundo Silva (1995b) e Silva \& Santos (2005). Entretanto, na APATM foram registradas 46 espécies que não foram observadas nas outras duas áreas. Algumas destas, como Penelope jacucaca e Picumnus pygmaeus, demonstram maior influência da fauna da Caatinga em parte da APATM, enquanto outras como Rostrhamus sociabilis, Aramus guarauna e Phimosus infuscatus indicam a presença de grandes corpos d'água, que não são encontrados na EESGT. Por outro lado, no PEJ, foram registradas 30 espécies ainda exclusivas desta UC, entre elas o globalmente ameaçado Harpyhaliaetus coronatus.

$\mathrm{O}$ alto número de espécies registrado nas três UCs indica que a região ainda é muito preservada, mantendo populações de espécies endêmicas e ameaçadas de extinção. Além disso, as três áreas, em conjunto, formam um mosaico de unidades de conservação fundamentais para a conservação da porção norte do Cerrado.

\section{Agradecimentos}

O levantamento de aves da EESGT fez parte do projeto "Inventário e zoneamento de vertebrados da Estação Ecológica Serra Geral do Tocantins: subsídios ao plano de manejo", financiado pela Fundação O Boticario de Proteção à Natureza (200710116). Ao Instituto Pequi - Pesquisa e Conservação do Cerrado e Conservação Internacional do Brasil, pelo apoio logístico. Somos gratos ao IBAMA e ICMBio pelo apoio às nossas pesquisas e licenças de coleta. VQP agradece à Fundação de Amparo à Pesquisa do Estado de São Paulo (FAPESP) pela bolsa de doutorado. LFS recebe bolsa de produtividade em pesquisa do CNPq. Às equipes de Herpetologia, Ictiologia e Mastozoologia e auxiliares de campo, pelo auxílio nas atividades dentro da EESGT. Cristiano Nogueira, pelo indispensável 
apoio em campo e pela leitura crítica do manuscrito. Aos dois revisores anônimos pelas valiosas sugestões e criticas feitas à este artigo.

\section{Referências Bibliográficas}

AB'SABER, A.N. 1977. Os domínios morfoclimáticos na América do Sul. Primeira aproximação. Geomorfologia. 52:1-18.

BAGNO, M.A. \& RODRIGUES, F.H.G. 1998. Novos registros de aves para o Estado de Goiás, Brasil. Ararajuba 6:64-65.

BRAZ, V.S. 2003. A representatividade das Unidades de Conservação do Cerrado na preservação da avifauna. Dissertação de mestrado, Universidade de Brasília, Brasília.

BRAZ, V.S., ABREU, T.L.S., LOPES, F.E., LEITE, L.O., FRANÇA, F.G.R., VASCONCELOS, M.M. \& BALBINO, S.F. 2003. Brazilian Merganser Mergus octosetaceus discovered in Jalapão State Park, Tocantins, Brazil. Cotinga 20:68-71.

BRASIL. Ministério do Meio Ambiente. Decreto 27/09/2001. Cria a Estação Ecológica Serra Geral do Tocantins, nos Estados do Tocantins e da Bahia, e dá outras providências. http://www.ibama.gov.br/siucweb/ listaUcCategoria.php?abrev=ESEC (último acesso em 12/06/2009).

COLLAR, N.J., GONZAGA, L.P., KRABBE, N., MADROÑO NIETO, NARANJO, G.A., PARKER III \& WEGE, D.C. 1992. Threatened birds of the America: The ICBP/IUCN Red Data Book. International Council for Bird Preservation, Cambridge.

COMITÊ BRASILEIRO DE REGISTROS ORNITOLÓGICOS - CRBO. 2009. Listas das aves do Brasil. Versão 9/8/2009. http://www.cbro. org.br

CORY, C.B. 1918. Catalogue of Birds of the Americas. Part II. Field Mus. Nat. Hist. Zool. Ser. 12(1):1-315.

HELLMAYR, C.E. 1929. A Contribution to the Ornithology of Northeastern Brazil. Field Mus. Nat. Hist. Zool. Ser. Publ. 12(18):235-501.

HELLMAYR, C.E. 1938. Catalogue of birds of the Americas, Part. XI. Field Mus. Nat. Hist. Zool. Ser. 13:1-430.

INSTITUTO BRASILEIRO DE GEOGRAFIA E ESTATÍSTICA - IBGE. 2006. Mapas de unidades de relevo do Brasil. $2^{\text {nd }}$ ed. IBGE.

INTERNATIONAL UNION FOR CONSERVATION OF NATURE - IUCN 2009. IUCN red list of threatened species. Version 2009.1. www. iucnredlist.org (ultimo acesso em 01/06/2009).

LEITE, L.O. \& LOPES, L.E. 2002. Fauna: Aves. pp. 63-67. In Plano de desenvolvimento sustentável para o entorno do Parque Estadual do Jalapão (S.R Silva \& P.G.P. Pereira, coords.). Conservation International do Brasil S/C. 190p.

LENCIONI NETO, F. 1995. Um híbrido entre Picumnus cirratus temminckii e P. albosquamatus guttifer (Piciformes: Picidae). Ararajuba 3:68-69.

LIMA, F.C.T. 1999. A range extension for the Caatinga Black-tyrant, Knipolegus franciscanus (Tyrannidae), a rare Brazilian endemic. Bull. Brit. Orn. Cl. 119(4):270-271.

LOPES, L.E. 2005. Field identification and new site records of Chapada Flycatcher Suiriri islerorum. Cotinga 24:38-41.

LOPES, L.E. 2007. Onthe range of the Lesser Kiskadee Philohydor lictor (Aves: Tyrannidae) in central-eastern Brazil. Ararajuba 15(3):433-435.

LOPES, L.E. 2008. The range ofthe Curl-crested Jay: lessons for evaluating bird endemism in South American Cerrado. Divers. Distrib. 14:561568. doi:10.1111/j.1472-4642.2007.00441.x

LOPES, L.E. \& MARINI, M.A. 2005. Biologia reprodutiva de Suiriri affinis e S. islerorum (Aves: Tyrannidae) no Cerrado do Brasil Central. Pap. Avulsos Zool. 45(12):127-141. doi: 10.1590/S0031-10492005001200001.

LOPES, L.E., MALACCO, G.B, VASCONCELOS, M.F., CARVALHO, C.E.A., DUCA, C., FERNANDES, A.M., D'ANGELO NETO, S. \& MARINI, M.A. 2008. Aves da região de Unaí e Cabeceira Grande, noroeste de Minas Gerais, Brasil. Rev. Bras. Ornitol.16:193-206.
MACHADO, A.B.M., DRUMMOND, G.M. \& PAGLIA, A.P. eds. 2008. Livro vermelho da fauna brasileira ameaçada de extinção. Ministério do Meio Ambiente, Brasília; Fundação Biodiversitas, Belo Horizonte, v. 2, p.1420. Biodiversidade, 19.

MAMEDE, F., GARCIA, P.Q. \& SOUSA JÚNIOR, W.C. 2002. Análise da viabilidade sócio-econômico-ambiental da transposição de águas da bacia do rio Tocantins para o rio São Francisco na região do Jalapão/ TO. Relatório Final. http://conservation-strategy.org/sites/default/files/ field-file/Jalapao_final_report.pdf (último acesso em 10/06/2009).

NAUMBURG, E.M.B. 1928. Remarks on Kaempfer's collections in eastern Brazil. Auk 45(1):60-65.

NAUMBURG, E.M.B. 1935. Gazetteer and maps showing collecting stations visited by Emil Kaempfer in eastern Brazil and Paraguay. Bull. Amer. Mus. Nat. Hist. 68:449-469.

PACHECO, J.F. 2004. As aves da Caatinga: uma análise histórica do conhecimento. In Biodiversidade da Caatinga: áreas e ações prioritárias para conservação. (J.M.C. Silva, M. Tabarelli, M.T. Fonseca \& L.V. Lins, eds.). Ministério do Meio Ambiente, Brasília, p. 189-250.

PACHECO, J.F. \& OLMOS, F. 2006. As aves do Tocantins 1: região sudeste. Ararajuba 14:55-71.

PACHECO, J.F. \& SILVA, R.S. 2002. The Brazilian Merganser Mergus octosetaceus in Jalapão, Tocantins, Brazil: results of a preliminary survey. Bird Life International - Brasil Program, São Paulo.

PAYNTER JUNIOR, R.A. \& TRAYLOR, M.A. 1991. Ornithological gazetteer of Brazil. Harvard University Press, Cambridge.

PINHEIRO, R.T. \& DORNAS, T. 2009. Distribuição e conservação das aves na região do Cantão, Tocantins: Ecótono Amazônia/cerrado. Biota Neotrop. 9(1): http://www.biotaneotropica.org.br/v9n1/pt/abstract?inv entory+bn02609012009 (último acesso em 12/06/2009).

PINTO, O.M.O. 1964. Ornitologia Brasiliense. Catalogo Descritivo e Ilustrado das Aves do Brasil. Departamento de Zoologia, Secretaria de Agricultura, São Paulo, v.1, 196 p.

RATTER, J.A., RIBEIRO, J.F. \& BRIDGWATER, S. 1997. The Brazilian cerrado vegetation and threats to its biodiversity. Ann. Bot. 80:223-270. doi:10.1006/anbo.1997.0469

RIDGELY, R.S. \& TUDOR, G. 1989. The birds of South America. v. 1. The Oscine Passerines. University Press, Oxford.

RIDGELY, R.S. \& TUDOR, G. 1994. The birdsof South America. v. 2. The Suboscine Passerines. University Press, Oxford.

SANTOS, M.P.D. 2001. Composição da avifauna nas Áreas de Proteção Ambiental Serra da Tabatinga e Chapada das Mangabeiras (PI, TO e MA). Bol. Mus. Para. E. Goeldi, Zool. 17(1):43-67.

TOCANTINS (Estado). Secretaria de Estado de Planejamento e Desenvolvimento Econômico - SEPLAN. 2003. Plano de Manejo do Parque Estadual do Jalapão. Palmas, p.132.

SHORT, L.L. 1982. Woodpeckers of the world. Delaware Museum of Natural History, Delaware. Monogr. Ser. 4.

SICK, H. 1997. Ornitologia brasileira. Edição revista e ampliada por José Fernando Pacheco. Editora Nova Fronteira, Rio de Janeiro.

SILVA, J.M.C. 1995a. Avian inventory of the cerrado region, South América: implications for biological conservation. Bird Cons. Intern. 5:291-304

SILVA, J.M.C. 1995b. Birds of the cerrado region. South America. Steenstrupia 21:69-72.

SILVA, J.M.C. 1997. Endemic bird species and conservation in the Cerrado region. South America. Biodiv. Conserv. 6:435-450. doi:10.1023/A:1018368809116

SILVA, J.M.C. \& SANTOS, M.P.D. 2005. A Importância relativa dos processos biogeográficos na formação da avifauna do Cerrado e de outros biomas brasileiros. In Biodiversidade Ecologia e Conservação do Cerrado (A.O. Scariot, J.C.S. Silva, Felfili \& J. Maria, org.). Ministério do Meio Ambiente, Brasília, p. 224-233

SILVEIRA, L.F. 1998. The birdsof Serra da Canastra National Park and adjacent areas, Minas Gerais, Brazil. Cotinga 10:55-63. 
SILVEIRA, L.F. 2008a. Taoniscus nanus. In Livro vermelho da fauna brasileira ameaçada de extinção. (A.B.M. Machado, G.M. Drummond \& A.P. Paglia, eds.). Ministério do Meio Ambiente, Brasília; Fundação Biodiversitas, Belo Horizonte, 2v., p.1420. Biodiversidade, 19.

SILVEIRA, L.F. 2008b. Nothura minor. In Livro vermelho da fauna brasileira ameaçada de extinção. (A.B.M. Machado, G.M. Drummond \& A.P. Paglia, eds.). Ministério do Meio Ambiente, Brasília; Fundação Biodiversitas, Belo Horizonte, 2v., p.1420. Biodiversidade, 19.

SILVEIRA, L.F. \& SILVEIRA, V.J. 1998.The biology of Dwarf Tinamou Taoniscus nanus, with notes on its breeding in captivity. Cotinga 9:42-46.
SNOW, D.W. 1982. The cotingas. British Museum (Natural History), London; Oxford University Press Oxford.

STOTZ, D.F., FITZPATRIC, J.W., PARKER III, T.A. \& MOSKOVITS, D.K. 1996. Neotropical birds: Ecology and conservation. University of Chicago Press, Chicago.

WINKLER, H. \& CHRISTIE, D.A. 2002. Family Picidae (woodpeckers). In Handbook of the Birds of the World, v.7, Jacamars to Woodpeckers (J. del Hoyo, A. Elliot, \& J. Sargatal, eds.). Lynx Editions, Barcelona.

ZIMMER, K.J., WHITTAKER, A \& OREN, D.C. 2001. A cryptic new species of flycatcher (Tyrannidae: Suiriri) from the Cerrado region of central South America. Auk118(1):56-78. doi:10.1642/00048038(2001)118[0056:ACNSOF]2.0.CO;2

Recebido em 12/05/2010

Versão reformulada recebida em 06/07/2010

Publicado em 21/03/2011 


\section{Apêndice 1}

Apêndice 1. Lista das espécies de aves registradas na Estação Ecológica da Serra Geral do Tocantins, no Parque Estadual do Jalapão (PEJ; Pacheco \& Silva 2002, Leite \& Lopes 2002, Braz 2003, Tocantins 2003) e nas Áreas de Proteção Ambiental Serra de Tabatinga e Chapada das Mangabeiras (APA; Santos 2001). Taxonomia segundo CBRO (Comitê... 2009). REG.: Forma como cada espécie foi registrada na EESG: COL: espécies coletadas; O: registros visuais; AU: registros auditivos. Espécies marcadas com um asterisco (*) foram também documentadas através de gravação em áudio. Localidades na EESG: 1. EESEC

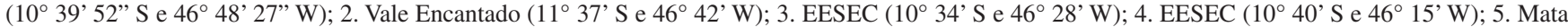

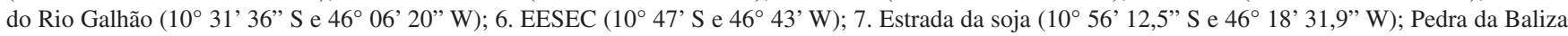
$\left(10^{\circ} 38^{\prime} \mathrm{S}\right.$ e $\left.46^{\circ} 07^{\prime} \mathrm{W}\right)$.

Appendix 1. Species recorded at Estação Ecológica da Serra Geral do Tocantins, Parque Estadual do Jalapão (PEJ; Pacheco \& Silva 2002, Leite \& Lopes 2002, Braz 2003, Tocantins 2003) and Áreas de Proteção Ambiental Serra de Tabatinga e Chapada das Mangabeiras (APA; Santos 2001). Taxonomy follows CBRO (Comitê... 2009). REG.: Documentation of the records at EESG: COL: collected; O: sight records; AU: heard only. Species with asterisk (*) were also

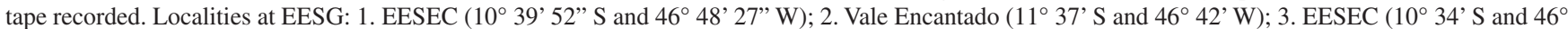

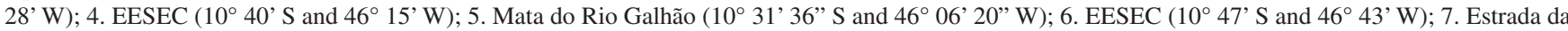
soja $\left(10^{\circ} 56^{\prime} 12,5^{\prime \prime} \mathrm{S}\right.$ and $\left.46^{\circ} 18^{\prime} 31,9^{\prime \prime} \mathrm{W}\right)$; Pedra da Baliza $\left(10^{\circ} 38^{\prime} \mathrm{S}\right.$ and $\left.46^{\circ} 07^{\prime} \mathrm{W}\right)$.

\begin{tabular}{|c|c|c|c|c|}
\hline Táxon & Nome em português & REG & Localidade na EESG & UCs adjacentes \\
\hline \multicolumn{5}{|l|}{ Struthioniformes } \\
\hline \multicolumn{5}{|l|}{ Rheidae } \\
\hline Rhea americana & Ema & $\mathrm{O}$ & $1,3,6$ & APA, PEJ \\
\hline \multicolumn{5}{|l|}{ Tinamiformes } \\
\hline \multicolumn{5}{|l|}{ Tinamidae } \\
\hline Crypturellus soui & Tururim & AU & $2,4,6$ & - \\
\hline Crypturellus undulatus* & Jaó & $\mathrm{COL}$ & $1,3,6$ & APA, PEJ \\
\hline Crypturellus tataupa & Inhambu-chintã & - & - & APA \\
\hline Crypturellus parvirostris* & Inhambu-chororó & $\mathrm{O}$ & $1,2,3,4,5,6$ & APA, PEJ \\
\hline Rhynchotus rufescens* & Perdiz & $\mathrm{COL}$ & $1,2,3,4,5,6$ & APA, PEJ \\
\hline Nothura maculosa & Codorna-do-nordeste & $\mathrm{COL}$ & $1,2,3,4,6,7$ & PEJ \\
\hline Nothura boraquira & Codorna-amarela & - & - & APA \\
\hline Taoniscus nanus & Inhambu-carapé & $\mathrm{O}$ & $1,3,6$ & PEJ \\
\hline \multicolumn{5}{|l|}{ Anseriformes } \\
\hline \multicolumn{5}{|l|}{ Anhimidae } \\
\hline Anhima cornuta & Anhuma & - & - & APA \\
\hline \multicolumn{5}{|l|}{ Anatidae } \\
\hline Dendrocygna viduata & Irerê & $\mathrm{O}$ & 3,6 & APA, PEJ \\
\hline Dendrocygna autumnalis & Asa-branca & - & - & APA \\
\hline Cairina moschata & Pato-do-mato & $\mathrm{O}$ & 3 & PEJ \\
\hline Sarkidiornis sylvicola & Pato-de-crista & $\mathrm{COL}$ & $2,3,4,6,7$ & APA \\
\hline Amazonetta brasiliensis & Pé-vermelho & - & - & APA, PEJ \\
\hline Netta erythrophthalma & Paturi-preta & - & - & APA \\
\hline Mergus octosetaceus & Pato-mergulhão & $\mathrm{O}$ & Nogueira, com. pess. & PEJ \\
\hline Nomonyx dominica & Marreca-de-bico-roxo & - & - & APA \\
\hline \multicolumn{5}{|l|}{ Galliformes } \\
\hline \multicolumn{5}{|l|}{ Cracidae } \\
\hline Ortalis superciliaris & Aracuã-de-sobrancelhas & - & - & APA \\
\hline Penelope superciliaris & Jacupemba & $\mathrm{COL}$ & $1,3,6$ & APA, PEJ \\
\hline Penelope jacuсаса & Jacucaca & - & - & APA \\
\hline Crax fasciolata & Mutum-de-penacho & - & - & PEJ \\
\hline \multicolumn{5}{|l|}{ Podicipediformes } \\
\hline \multicolumn{5}{|l|}{ Podicipedidae } \\
\hline Tachybaptus dominicus & Mergulhão-pequeno & - & - & APA, PEJ \\
\hline Podilymbus podiceps & Mergulhão-caçador & - & - & APA, PEJ \\
\hline \multicolumn{5}{|l|}{ Pelecaniformes } \\
\hline \multicolumn{5}{|l|}{ Phalacrocoracidae } \\
\hline Phalacrocorax brasilianus & Biguá & $\mathrm{O}$ & 1 & APA, PEJ \\
\hline \multicolumn{5}{|l|}{ Anhingidae } \\
\hline Anhinga anhinga & Biguatinga & $\mathrm{O}$ & 1 & PEJ \\
\hline
\end{tabular}


Apêndice 1. Continuação...

\begin{tabular}{|c|c|c|c|c|}
\hline Táxon & Nome em português & REG & Localidade na EESG & UCs adjacentes \\
\hline \multicolumn{5}{|l|}{ Ciconiiformes } \\
\hline \multicolumn{5}{|l|}{ Ardeidae } \\
\hline Tigrisoma lineatum & Socó-boi & $\mathrm{O}$ & 3 & APA, PEJ \\
\hline Nycticorax nycticorax & Savacu & - & - & APA \\
\hline Ixobrychus exilis & Socoí-vermelho & - & - & APA \\
\hline Butorides striata & Socozinho & $\mathrm{COL}$ & $1,2,3$ & APA, PEJ \\
\hline Bubulcus ibis & Garça-vaqueira & $\mathrm{O}$ & 3 & APA, PEJ \\
\hline Ardea cocoi & Garça-moura & - & - & PEJ \\
\hline Ardea alba & Garça-branca-grande & $\mathrm{O}$ & 3 & APA, PEJ \\
\hline Syrigma sibilatrix & Maria-faceira & $\mathrm{O}$ & $2,4,6$ & - \\
\hline Egretta thula & Garça-branca-pequena & - & - & APA, PEJ \\
\hline \multicolumn{5}{|l|}{ Cochleariidae } \\
\hline Cochlearius cochlarius & Arapapá & - & - & APA \\
\hline \multicolumn{5}{|l|}{ Threskiornithidae } \\
\hline Mesembrinibis cayennensis & Coró-coró & $\mathrm{O}$ & 3 & PEJ \\
\hline Phimosus infuscatus & Tapicuru-de-cara-pelada & - & - & APA \\
\hline Theristicus caudatus* & Curicaca & $\mathrm{O}$ & $1,2,3,5$ & APA, PEJ \\
\hline \multicolumn{5}{|l|}{ Ciconiidae } \\
\hline Mycteria americana & Cabeça-seca & - & - & PEJ \\
\hline \multicolumn{5}{|l|}{ Cathartiformes } \\
\hline \multicolumn{5}{|l|}{ Cathartidae } \\
\hline Cathartes aura & Urubu-de-cabeça-vermelha & $\mathrm{COL}$ & $1,3,4,5,6$ & APA, PEJ \\
\hline Cathartes burrovianus & Urubu-de-cabeça-amarela & $\mathrm{COL}$ & $1,3,4,5,6$ & APA, PEJ \\
\hline Coragyps atratus & Urubu-de-cabeça-preta & $\mathrm{O}$ & $1,3,5,6$ & APA, PEJ \\
\hline Sarcoramphus papa & Urubu-rei & $\mathrm{O}$ & $1,2,4$ & APA, PEJ \\
\hline \multicolumn{5}{|l|}{ Falconiformes } \\
\hline \multicolumn{5}{|l|}{ Accipitridae } \\
\hline Chondrohierax uncinatus & Caracoleiro & COL & $2,4,5,6$ & - \\
\hline Elanoides forficatus & Gavião-tesoura & $\mathrm{O}$ & 1,3 & APA, PEJ \\
\hline Gampsonyx swainsonii & Gaviãozinho & - & - & APA, PEJ \\
\hline Elanus leucurus & Gavião-peneira & $\mathrm{O}$ & 3,6 & APA, PEJ \\
\hline Rostrhamus sociabilis & Gavião-caramujeiro & - & - & APA \\
\hline Ictinia plumbea & Sovi & $\mathrm{O}$ & $2,4,6$ & PEJ \\
\hline Accipiter striatus & Gavião-miúdo & $\mathrm{COL}$ & 1,3 & - \\
\hline Buteogallus urubitinga & Gavião-preto & $\mathrm{O}$ & 1 & PEJ \\
\hline Heterospizias meridionalis & Gavião-caboclo & $\mathrm{O}$ & $1,2,3,4,5,6$ & APA, PEJ \\
\hline Rupornis magnirostris & Gavião-carijó & $\mathrm{COL}$ & $1,2,3,4,5,6$ & APA, PEJ \\
\hline Buteo albicaudatus & Gavião-de-rabo-branco & $\mathrm{COL}$ & $1,2,3,4,5,6$ & APA, PEJ \\
\hline Buteo melanoleucus & Águia-chilena & $\mathrm{COL}$ & 3,4 & APA, PEJ \\
\hline Buteo nitidus & Gavião-pedrês & $\mathrm{O}$ & 3,6 & PEJ \\
\hline Busarellus nigricollis & Gavião-belo & - & - & PEJ \\
\hline Harpyhaliaetus coronatus & Águia-cinzenta & - & - & PEJ \\
\hline Geranospiza caerulescens & Gavião-pernilongo & - & - & APA, PEJ \\
\hline Spizaetus melanoleucus & Gavião-pato & $\mathrm{O}$ & 5 & - \\
\hline \multicolumn{5}{|l|}{ Falconidae } \\
\hline Caracara plancus & Caracará & $\mathrm{COL}$ & $1,2,3,5,6,8$ & APA, PEJ \\
\hline Ibycter americanus & Gralhão & - & - & PEJ \\
\hline Milvago chimachima & Carrapateiro & $\mathrm{COL}$ & $1,2,3,5,6$ & APA, PEJ \\
\hline Herpetotheres cachinnans & Acauã & $\mathrm{O}$ & $1,3,6$ & APA, PEJ \\
\hline Falco sparverius & Quiriquiri & COL & $1,2,3,4,5,6$ & APA, PEJ \\
\hline Falco rufigularis & Cauré & $\mathrm{O}$ & 1 & APA, PEJ \\
\hline
\end{tabular}


Apêndice 1. Continuação...

\begin{tabular}{|c|c|c|c|c|}
\hline Táxon & Nome em português & REG & Localidade na EESG & UCs adjacentes \\
\hline Falco femoralis & Falcão-de-coleira & COL & $1,2,3,4,5,6,7$ & APA, PEJ \\
\hline \multicolumn{5}{|l|}{ Gruiformes } \\
\hline \multicolumn{5}{|l|}{ Aramidae } \\
\hline Aramus guarauna & Carão & - & - & APA \\
\hline \multicolumn{5}{|l|}{ Rallidae } \\
\hline Aramides cajanea & Saracura-três-potes & $\mathrm{O}$ & 1,2 & APA, PEJ \\
\hline Laterallus viridis & Sanã-castanha & $\mathrm{COL}$ & 3,5 & PEJ \\
\hline Laterallus melanophaius & Sanã-parda & - & - & APA \\
\hline Porzana albicollis & Sanã-carijó & $\mathrm{AU}$ & 2,3 & PEJ \\
\hline Gallinula chloropus & Frango-d'água-comum & $\mathrm{O}$ & 3 & APA \\
\hline Porphyrio martinica & Frango-d'água-azul & - & - & APA \\
\hline \multicolumn{5}{|l|}{ Cariamidae } \\
\hline Cariama cristata* & Seriema & $\mathrm{O}$ & $1,2,3,4,5,6$ & APA, PEJ \\
\hline \multicolumn{5}{|l|}{ Charadriiformes } \\
\hline \multicolumn{5}{|l|}{ Charadriidae } \\
\hline Vanellus chilensis & Batuíra-de-esporão & $\mathrm{COL}$ & $1,3,4,5,6$ & APA, PEJ \\
\hline Vanellus cayanus & Quero-quero & - & - & APA, PEJ \\
\hline Charadrius cf. wilsonia & Batuíra-bicuda & - & - & PEJ \\
\hline \multicolumn{5}{|l|}{ Recurvirostridae } \\
\hline Himantopus himantopus & - & - & - & APA \\
\hline \multicolumn{5}{|l|}{ Scolopacidae } \\
\hline Actitis macularius & Maçarico-pintado & - & - & APA \\
\hline Gallinago undulata & Narcejão & $\mathrm{AU}$ & $1,3,6$ & APA \\
\hline Tringa solitaria & Maçarico-solitário & COL & 3,6 & - \\
\hline \multicolumn{5}{|l|}{ Jacanidae } \\
\hline Jacana jacana & Jaçanã & $\mathrm{O}$ & 1,3 & APA, PEJ \\
\hline \multicolumn{5}{|l|}{ Columbiformes } \\
\hline \multicolumn{5}{|l|}{ Columbidae } \\
\hline Columbina minuta & Rolinha-de-asa-canela & $\mathrm{O}$ & $1,2,3,4,6$ & APA, PEJ \\
\hline Columbina talpacoti & Rolinha-roxa & $\mathrm{O}$ & $1,3,5$ & APA, PEJ \\
\hline Columbina squammata* & Fogo-apagou & $\mathrm{COL}$ & $1,3,5$ & APA, PEJ \\
\hline Columbina picui & Rolinha-picui & $\mathrm{O}$ & 3 & APA, PEJ \\
\hline Uropelia campestris & Rolinha-vaqueira & $\mathrm{COL}$ & $1,2,3,5,6$ & PEJ \\
\hline Claravis pretiosa & Pararu-azul & - & - & APA \\
\hline Columba livia & Pombo-doméstico & - & - & APA \\
\hline Patagioenas speciosa & Pomba-trocal & $\mathrm{O}$ & 1 & - \\
\hline Patagioenas picazuro & Pombão & $\mathrm{O}$ & $1,2,3,5,6$ & APA, PEJ \\
\hline Patagioenas cayennensis & Pomba-galega & $\mathrm{COL}$ & $1,2,3,5,6$ & PEJ \\
\hline Patagioenas plumbea & Pomba-amargosa & $\mathrm{O}$ & $2,4,6$ & PEJ \\
\hline Zenaida auriculata & Pomba-de-bando & $\mathrm{O}$ & $1,2,4,6$ & APA, PEJ \\
\hline Leptotila verreauxi* & Juriti-pupu & $\mathrm{O}$ & 1 & APA, PEJ \\
\hline Leptotila rufaxilla* & Juriti-gemedeira & COL & $1,3,6$ & PEJ \\
\hline \multicolumn{5}{|l|}{ Psittaciformes } \\
\hline \multicolumn{5}{|l|}{ Psittacidae } \\
\hline Anodorhynchus hyacinthinus & Arara-azul-grande & $\mathrm{O}$ & $1,3,6$ & APA, PEJ \\
\hline Ara ararauna* & Arara-canindé & $\mathrm{COL}$ & $1,3,6$ & APA, PEJ \\
\hline Ara chloropterus & Arara-vermelha-grande & $\mathrm{O}$ & 1 & APA, PEJ \\
\hline Orthopsittaca manilata & Maracanã-do-buriti & $\mathrm{COL}$ & 1,3 & PEJ \\
\hline Primolius maracana & Maracanã-verdadeira & - & - & APA \\
\hline Diopsittaca nobilis & Maracanã-pequena & $\mathrm{O}$ & 1 & APA, PEJ \\
\hline Aratinga acuticaudata* & Aratinga-de-testa-azul & $\mathrm{COL}$ & $1,2,3,4,5,6$ & APA, PEJ \\
\hline
\end{tabular}


Apêndice 1. Continuação...

\begin{tabular}{|c|c|c|c|c|}
\hline Táxon & Nome em português & REG & Localidade na EESG & UCs adjacentes \\
\hline Aratinga leucophthalma & Periquitão-maracanã & $\mathrm{O}$ & 3 & PEJ \\
\hline Aratinga jandaya & Jandaia-verdadeira & - & - & APA, PEJ \\
\hline Aratinga aurea* & Periquito-rei & $\mathrm{COL}$ & $1,2,3,4,5,6$ & APA, PEJ \\
\hline Forpus xanthopterygius & Tuim & - & - & APA, PEJ \\
\hline Brotogeris chiriri & Periquito-de-encontro-amarelo & $\mathrm{COL}$ & $1,3,6$ & APA, PEJ \\
\hline Alipiopsitta xanthops* & Papagaio-galego & COL & 1,5 & APA, PEJ \\
\hline Pionus menstruus & Maitaca-de-cabeça-azul & $\mathrm{O}$ & 3,6 & - \\
\hline Pionus maximiliani & Maitaca-verde & - & - & APA \\
\hline Amazona aestiva & Papagaio-verdadeiro & COL & $1,2,3,5,6$ & APA, PEJ \\
\hline Amazona amazonica* & Curica & $\mathrm{O}$ & 1 & APA \\
\hline \multicolumn{5}{|l|}{ Cuculiformes } \\
\hline \multicolumn{5}{|l|}{ Cuculidae } \\
\hline Piaya cayana & Alma-de-gato & $\mathrm{O}$ & $1,3,6$ & APA, PEJ \\
\hline Coccyzus melacoryphus & Papa-lagarta-acanelado & $\mathrm{O}$ & $2,4,6$ & - \\
\hline Crotophaga major & Anu-coroca & $\mathrm{O}$ & 3 & APA, PEJ \\
\hline Crotophaga ani & Anu-preto & $\mathrm{O}$ & 1,3 & APA, PEJ \\
\hline Guira guira & Anu-branco & $\mathrm{O}$ & $1,2,3,5$ & APA, PEJ \\
\hline Tapera naevia & Saci & $\mathrm{O}$ & 1,3 & APA, PEJ \\
\hline Dromococcyx phasianellus & Peixe-frito-verdadeiro & - & - & APA \\
\hline \multicolumn{5}{|l|}{ Strigiformes } \\
\hline \multicolumn{5}{|l|}{ Tytonidae } \\
\hline Tyto alba & Coruja-da-igreja & $\mathrm{O}$ & 7 & APA, PEJ \\
\hline \multicolumn{5}{|l|}{ Strigidae } \\
\hline Megascops choliba & Corujinha-do-mato & $\mathrm{AU}$ & 1 & APA, PEJ \\
\hline Bubo virginianus & Jacurutu & - & - & PEJ \\
\hline Strix huhula & Coruja-preta & - & - & APA \\
\hline Glaucidium brasilianum* & Caburé & $\mathrm{COL}$ & 1,5 & APA, PEJ \\
\hline Athene cunicularia & Coruja-buraqueira & $\mathrm{COL}$ & $1,2,3,5,6$ & APA, PEJ \\
\hline Asio clamator & Coruja-orelhuda & COL & 1 & PEJ \\
\hline Asio flammeus & Mocho-dos-banhados & $\mathrm{COL}$ & 7 & - \\
\hline \multicolumn{5}{|l|}{ Caprimulgiformes } \\
\hline \multicolumn{5}{|l|}{ Nyctibiidae } \\
\hline Nyctibius griseus & Mãe-da-lua & AU & 1 & PEJ \\
\hline \multicolumn{5}{|l|}{ Caprimulgidae } \\
\hline Lurocalis semitorquatus & Tuju & $\mathrm{AU}$ & 6 & PEJ \\
\hline Chordeiles pusillus* & Bacurauzinho & $\mathrm{COL}$ & $1,3,5,6$ & PEJ \\
\hline Podager nacunda & Corucão & $\mathrm{O}$ & 1 & APA, PEJ \\
\hline Nyctidromus albicollis* & Bacurau & $\mathrm{COL}$ & $1,3,5$ & APA, PEJ \\
\hline Caprimulgus rufus & João-corta-pau & $\mathrm{AU}$ & 1 & PEJ \\
\hline Caprimulgus longirostris & Bacurau-da-telha & $\mathrm{COL}$ & 5 & - \\
\hline Caprimulgus parvulus & Bacurau-chintã & - & - & PEJ \\
\hline Hydropsalis torquata & Bacurau-tesoura & $\mathrm{COL}$ & $1,3,4,5,6$ & APA, PEJ \\
\hline \multicolumn{5}{|l|}{ Apodiformes } \\
\hline \multicolumn{5}{|l|}{ Apodidae } \\
\hline Streptoprocne zonaris & Taperuçu-de-coleira-branca & - & - & APA, PEJ \\
\hline Cypseloides senex & Taperuçu-velho & - & - & PEJ \\
\hline Cypseloides sp. & - & $\mathrm{O}$ & 1 & - \\
\hline Chaetura meridionalis & Andorinhão-do-temporal & $\mathrm{O}$ & $1,3,5,6$ & PEJ \\
\hline Tachornis squamata & Tesourinha & $\mathrm{COL}$ & $1,2,3,5,6$ & APA, PEJ \\
\hline \multicolumn{5}{|l|}{ Trochilidae } \\
\hline Phaethornis pretrei* & Rabo-branco-acanelado & $\mathrm{O}$ & $1,2,3,5$ & APA, PEJ \\
\hline
\end{tabular}


Apêndice 1. Continuação...

\begin{tabular}{|c|c|c|c|c|}
\hline Táxon & Nome em português & REG & Localidade na EESG & UCs adjacentes \\
\hline Phaethornis ruber & Rabo-branco-rubro & - & - & PEJ \\
\hline Campylopterus largipennis & Asa-de-sabre-cinza & - & - & APA \\
\hline Eupetomena macroura & Beija-flor-tesoura & $\mathrm{COL}$ & $1,2,3,4,5,6$ & APA, PEJ \\
\hline Colibri serrirostris & Beija-flor-de-orelha-violeta & - & - & APA \\
\hline Anthracothorax nigricollis & Beija-flor-de-veste-preta & $\mathrm{COL}$ & 1,2 & - \\
\hline Chrysolampis mosquitus & Beija-flor-vermelho & $\mathrm{COL}$ & 1 & APA \\
\hline Chlorostilbon lucidus & Besourinho-de-bico-vermelho & $\mathrm{O}$ & $1,3,6$ & APA, PEJ \\
\hline Thalurania furcata & Beija-flor-tesoura-verde & $\mathrm{COL}$ & $1,2,3,5,6$ & PEJ \\
\hline Amazilia fimbriata & Beija-flor-de-garganta-verde & - & - & APA, PEJ \\
\hline Heliactin bilophus* & Chifre-de-ouro & $\mathrm{COL}$ & $1,2,3,5$ & APA, PEJ \\
\hline Calliphlox amethystina & Estrelinha-ametista & $\mathrm{O}$ & 1 & PEJ \\
\hline \multicolumn{5}{|l|}{ Trogoniformes } \\
\hline \multicolumn{5}{|l|}{ Trogonidae } \\
\hline Trogon curucui & Surucuá-de-barriga-vermelha & - & - & APA, PEJ \\
\hline \multicolumn{5}{|l|}{ Coraciiformes } \\
\hline \multicolumn{5}{|l|}{ Alcedinidae } \\
\hline Megaceryle torquata & Martim-pescador-grande & - & - & APA, PEJ \\
\hline Chloroceryle amazona & Martim-pescador-verde & $\mathrm{O}$ & $1,2,5$ & APA, PEJ \\
\hline Chloroceryle aenea & Martinho & $\mathrm{COL}$ & $1,2,5$ & - \\
\hline Chloroceryle americana & Martim-pescador-pequeno & $\mathrm{O}$ & 1,2 & APA, PEJ \\
\hline \multicolumn{5}{|l|}{ Momotidae } \\
\hline Momotus momota & Udu-de-coroa-azul & $\mathrm{O}$ & $2,4,6$ & PEJ \\
\hline \multicolumn{5}{|l|}{ Galbuliformes } \\
\hline \multicolumn{5}{|l|}{ Galbulidae } \\
\hline Galbula ruficauda & Ariramba-de-cauda-ruiva & $\mathrm{COL}$ & $1,2,3,5,6$ & APA, PEJ \\
\hline \multicolumn{5}{|l|}{ Bucconidae } \\
\hline Nystalus chacuru & João-bobo & $\mathrm{COL}$ & $1,2,3,4,5,6$ & APA, PEJ \\
\hline Nystalus maculatus & Rapazinho-dos-velhos & - & - & APA, PEJ \\
\hline Nonnula rubecula & Macuru & $\mathrm{COL}$ & 2 & APA \\
\hline Monasa nigrifrons & Chora-chuva-preto & $\mathrm{O}$ & 1 & PEJ \\
\hline Chelidoptera tenebrosa & Urubuzinho & - & - & PEJ \\
\hline \multicolumn{5}{|l|}{ Piciformes } \\
\hline \multicolumn{5}{|l|}{ Ramphastidae } \\
\hline Ramphastos toco & Tucanuçu & $\mathrm{O}$ & $1,3,6$ & APA, PEJ \\
\hline \multicolumn{5}{|l|}{ Picidae } \\
\hline Picumnus pygmaeus & Pica-pau-anão-pintado & - & - & APA \\
\hline Picumnus albosquamatus & Pica-pau-anão-escamado & $\mathrm{COL}$ & $2,4,6$ & PEJ \\
\hline Picumnus sp. & - & $\mathrm{COL}$ & 3,5 & - \\
\hline Melanerpes candidus & Birro, pica-pau-branco & $\mathrm{COL}$ & $1,2,5$ & APA, PEJ \\
\hline Veniliornis passerinus & Picapauzinho-anão & $\mathrm{COL}$ & 2,3 & APA \\
\hline Veniliornis mixtus & Pica-pau-chorão & - & - & PEJ \\
\hline Piculus chrysochloros & Pica-pau-dourado-escuro & $\mathrm{O}$ & 3,6 & - \\
\hline Colaptes melanochloros & Pica-pau-verde-barrado & $\mathrm{COL}$ & 3,6 & APA, PEJ \\
\hline Colaptes campestris & Pica-pau-do-campo & $\mathrm{COL}$ & $1,2,3,5,6$ & APA, PEJ \\
\hline Celeus flavescens* & Pica-pau-de-cabeça-amarela & $\mathrm{COL}$ & $3,5,6$ & APA, PEJ \\
\hline Celeus flavus & Pica-pau-amarelo & $\mathrm{O}$ & 3 & - \\
\hline Dryocopus lineatus & Pica-pau-de-banda-branca & $\mathrm{COL}$ & $1,3,5,6$ & APA, PEJ \\
\hline Campephilus melanoleucos & Pica-pau-de-topete-vermelho & $\mathrm{O}$ & $1,2,3$ & APA, PEJ \\
\hline \multicolumn{5}{|l|}{ Passeriformes } \\
\hline \multicolumn{5}{|l|}{ Melanopareiidae } \\
\hline Melanopareia torquata* & Tapaculo-de-colarinho & $\mathrm{COL}$ & $1,2,3,5,6$ & APA, PEJ \\
\hline
\end{tabular}


Rego, M.A. et al.

Apêndice 1. Continuação...

\begin{tabular}{|c|c|c|c|c|}
\hline Táxon & Nome em português & REG & Localidade na EESG & UCs adjacentes \\
\hline \multicolumn{5}{|l|}{ Thamnophilidae } \\
\hline Taraba major & Choró-boi & - & - & APA \\
\hline Thamnophilus pelzelni & Choca-do-planalto & $\mathrm{O}$ & $1,2,3$ & PEJ \\
\hline Thamnophilus doliatus & Choca-barrada & - & - & APA, PEJ \\
\hline Thamnophilus torquatus* & Choca-de-asa-vermelha & $\mathrm{COL}$ & 1,2 & APA, PEJ \\
\hline Herpsilochmus atricapillus* & Chorozinho-de-chapéu-preto & $\mathrm{COL}$ & 2 & APA, PEJ \\
\hline Herpsilochmus longirostris* & Chorozinho-de-bico-comprido & $\mathrm{COL}$ & $1,2,3,5,6$ & PEJ \\
\hline Formicivora grisea & Papa-formiga-pardo & $\mathrm{O}$ & 1,2 & APA, PEJ \\
\hline Formicivora rufa* & Papa-formiga-vermelho & $\mathrm{COL}$ & $1,3,5,6$ & APA, PEJ \\
\hline Formicivora melanogaster & Formigueiro-de-barriga-preta & - & - & PEJ \\
\hline \multicolumn{5}{|l|}{ Dendrocolaptidae } \\
\hline Sittasomus griseicapillus & Arapaçu-verde & COL & $1,3,5,6$ & APA, PEJ \\
\hline Dendrocolaptes platyrostris & Arapaçu-grande & $\mathrm{COL}$ & $3,5,6$ & APA, PEJ \\
\hline Xiphorhynchus picus & Arapaçu-de-bico-branco & $\mathrm{COL}$ & 1 & APA \\
\hline Xiphorhynchus guttatus & Arapaçu-de-garganta-amarela & $\mathrm{AU}$ & $2,4,6$ & PEJ \\
\hline Lepidocolaptes angustirostris & Arapaçu-de-cerrado & $\mathrm{COL}$ & $1,2,3,5,6$ & APA, PEJ \\
\hline Campylorhamphus trochilirostris & Arapaçu-beija-flor & - & - & APA \\
\hline \multicolumn{5}{|l|}{ Furnariidae } \\
\hline Furnarius leucopus & Casaca-de-couro-amarelo & - & - & APA \\
\hline Furnarius figulus & Casaca-de-couro-da-lama & - & - & APA \\
\hline Furnarius rufus & João-de-barro & $\mathrm{O}$ & 1 & PEJ \\
\hline Synallaxis frontalis* & Petrim & $\mathrm{COL}$ & $1,3,5,6$ & APA, PEJ \\
\hline Synallaxis albescens* & Uí-pi & $\mathrm{COL}$ & $1,2,3,5,6$ & APA, PEJ \\
\hline Synallaxis scutata & Estrelinha-preta & - & - & APA \\
\hline Certhiaxis cinnamomeus & Curutié & - & - & APA, PEJ \\
\hline Cranioleuca vulpina & Arredio-do-rio & - & - & APA \\
\hline Phacellodomus rufifrons & João-de-pau & COL & $1,4,5$ & APA, PEJ \\
\hline Phacellodomus ruber & Graveteiro & $\mathrm{O}$ & 1 & - \\
\hline Berlepschia rikeri* & Limpa-folha-do-buriti & COL & $1,2,3,5,6$ & APA, PEJ \\
\hline Xenops rutilans & Bico-virado-carijó & - & - & APA, PEJ \\
\hline \multicolumn{5}{|l|}{ Tyrannidae } \\
\hline Leptopogon amaurocephalus & Cabeçudo & $\mathrm{O}$ & 1 & APA, PEJ \\
\hline Corythopis delalandi & Estalador & - & - & PEJ \\
\hline Hemitriccus striaticollis* & Sebinho-rajado-amarelo & $\mathrm{COL}$ & $1,2,3,5,6$ & PEJ \\
\hline Hemitriccus margaritaceiventer* & Sebinho-de-olho-de-ouro & $\mathrm{O}$ & 1 & APA, PEJ \\
\hline Poecilotriccus latirostris & Ferreirinho-de-cara-parda & $\mathrm{O}$ & 5 & - \\
\hline Todirostrum cinereum & Ferreirinho-relógio & $\mathrm{COL}$ & $1,3,5,6$ & APA, PEJ \\
\hline Phyllomyias fasciatus & Piolhinho & $\mathrm{AU}$ & 1 & APA \\
\hline Myiopagis viridicata & Guaracava-de-crista-alaranjada & - & - & APA, PEJ \\
\hline Myiopagis gaimardii & Maria-pechim & $\mathrm{O}$ & 1,2 & PEJ \\
\hline Elaenia flavogaster* & Guaracava-de-barriga-amarela & $\mathrm{COL}$ & $1,3,5$ & APA, PEJ \\
\hline Elaenia parvirostris & Guaracava-de-bico-curto & - & - & PEJ \\
\hline Elaenia cristata & Guaracava-de-topete-uniforme & $\mathrm{COL}$ & $1,2,3,5,6$ & APA, PEJ \\
\hline Elaenia chiriquensis & Chibum & $\mathrm{COL}$ & $1,3,5,6$ & PEJ \\
\hline Camptostoma obsoletum & Risadinha & $\mathrm{COL}$ & $1,2,3,5$ & APA, PEJ \\
\hline Suiriri suiriri & Suiriri-cinzento & $\mathrm{COL}$ & $1,2,3,5,6$ & PEJ \\
\hline Suiriri islerorum & Suiriri-da-chapada & COL & $2,4,5,6$ & - \\
\hline Serpophaga nigricans & João-pobre & - & - & PEJ \\
\hline Phaeomyias murina & Bagageiro & $\mathrm{O}$ & 5 & APA \\
\hline Euscarthmus meloryphus & Barulhento & $\mathrm{O}$ & 1 & - \\
\hline Euscarthmus rufomarginatus* & Maria-corruíra & $\mathrm{COL}$ & $1,2,3,6$ & APA, PEJ \\
\hline
\end{tabular}


As aves da Estação Ecológica Serra Geral do Tocantins

Apêndice 1. Continuação...

\begin{tabular}{|c|c|c|c|c|}
\hline Táxon & Nome em português & REG & Localidade na EESG & UCs adjacentes \\
\hline Sublegatus modestus & Guaracava-modesta & $\mathrm{COL}$ & 1,3 & APA, PEJ \\
\hline Tolmomyias sulphurescens & Bico-chato-de-orelha-preta & - & - & PEJ \\
\hline Tolmomyias flaviventris & Bico-chato-amarelo & $\mathrm{COL}$ & $1,3,5,6$ & APA, PEJ \\
\hline Platyrhinchus mystaceus & Patinho & - & - & PEJ \\
\hline Myiophobus fasciatus & Filipe & $\mathrm{AU}$ & $1,3,5,6$ & PEJ \\
\hline Myiobius barbatus & Assanhadinho & - & - & APA \\
\hline Lathrotriccus euleri & Enferrujado & $\mathrm{COL}$ & 1,5 & PEJ \\
\hline Cnemotriccus fuscatus & Guaracavuçu & $\mathrm{COL}$ & 5 & APA, PEJ \\
\hline Contopus cinereus & Papa-moscas-cinzento & - & - & APA \\
\hline Pyrocephalus rubinus & Príncipe & - & - & APA, PEJ \\
\hline Knipolegus franciscanus & Maria-preta-do-nordeste & $\mathrm{COL}$ & 2 & - \\
\hline Xolmis cinereus & Primavera & $\mathrm{O}$ & $1,2,3,6$ & APA, PEJ \\
\hline Xolmis velatus & Noivinha-branca & COL & $1,3,4$ & PEJ \\
\hline Fluvicola albiventer & Lavadeira-de-cara-branca & - & - & PEJ \\
\hline Fluvicola nengeta & Lavadeira-mascarada & - & - & APA \\
\hline Arundinicola leucocephala & Freirinha & $\mathrm{O}$ & 1 & APA, PEJ \\
\hline Colonia colonus & Viuvinha & - & - & APA, PEJ \\
\hline Satrapa icterophrys & Suiriri-pequeno & - & - & APA, PEJ \\
\hline Hirundinea ferruginea & Gibão-de-couro & - & - & APA, PEJ \\
\hline Machetornis rixosa & Suiriri-cavaleiro & $\mathrm{O}$ & 2 & APA \\
\hline Legatus leucophaius & Bem-te-vi-pirata & $\mathrm{O}$ & 1 & PEJ \\
\hline Pitangus sulphuratus* & Bem-te-vi & $\mathrm{COL}$ & $1,2,3,5,6$ & APA, PEJ \\
\hline Philohydor lictor & Bentevizinho-do-brejo & $\mathrm{O}$ & 1 & APA, PEJ \\
\hline Myiodynastes maculatus & Bem-te-vi-rajado & AU & 1 & APA, PEJ \\
\hline Myiozetetes similis & Bentevizinho-de-penacho-vermelho & - & - & APA \\
\hline Myiozetetes cayanensis & Bentevizinho-de-asa-ferrugínea & - & - & APA, PEJ \\
\hline Megarynchus pitangua* & Neinei & $\mathrm{COL}$ & $1,2,3,5,6$ & APA, PEJ \\
\hline Tyrannopsis sulphurea & Suiriri-de-garganta-rajada & $\mathrm{COL}$ & 3,5 & - \\
\hline Empidonomus varius & Peitica & $\mathrm{O}$ & 1 & PEJ \\
\hline Griseotyrannus aurantioatrocristatus & Peitica-de-chapéu-preto & - & - & APA \\
\hline Tyrannus albogularis & Suiriri-de-garganta-branca & $\mathrm{COL}$ & $1,2,3,5,6$ & PEJ \\
\hline Tyrannus melancholicus & Suiriri & $\mathrm{O}$ & 1,5 & APA, PEJ \\
\hline Tyrannus savana & Tesourinha & $\mathrm{COL}$ & $1,2,3,5,6$ & APA \\
\hline Sirystes sibilator & Gritador & $\mathrm{O}$ & 1 & PEJ \\
\hline Casiornis rufus & Caneleiro & $\mathrm{O}$ & $2,4,6$ & - \\
\hline Casiornis fuscus & Caneleiro-enxofre & - & - & APA, PEJ \\
\hline Myiarchus swainsoni & Irré & $\mathrm{COL}$ & 1,2 & APA, PEJ \\
\hline Myiarchus ferox* & Maria-cavaleira & $\mathrm{COL}$ & 1,5 & APA, PEJ \\
\hline Myiarchus tyrannulus & Maria-cavaleira-de-rabo-enferrujado & $\mathrm{O}$ & $2,4,6$ & APA, PEJ \\
\hline \multicolumn{5}{|l|}{ Cotingidae } \\
\hline Procnias averano* & Araponga-do-nordeste & COL & 3,5 & APA \\
\hline \multicolumn{5}{|l|}{ Pipridae } \\
\hline Neopelma pallescens & Fruxu-do-cerradão & $\mathrm{AU}$ & $2,4,6$ & APA, PEJ \\
\hline Antilophia galeata & Soldadinho & $\mathrm{COL}$ & $1,2,3,5,6$ & APA, PEJ \\
\hline Pipra fasciicauda & Uirapuru-laranja & - & - & PEJ \\
\hline \multicolumn{5}{|l|}{ Tityridae } \\
\hline Tityra inquisitor & Anambé-branco-de-bochecha-parda & - & - & APA, PEJ \\
\hline Tityra cayana & Anambé-branco-de-rabo-preto & $\mathrm{COL}$ & 3,5 & APA, PEJ \\
\hline Pachyramphus viridis & Caneleiro-verde & - & - & APA, PEJ \\
\hline Pachyramphus validus & Caneleiro-de-chapéu-preto & - & - & APA, PEJ \\
\hline Pachyramphus polychopterus & Caneleiro-preto & $\mathrm{AU}$ & 3 & APA, PEJ \\
\hline
\end{tabular}


Rego, M.A. et al

Apêndice 1. Continuação...

\begin{tabular}{|c|c|c|c|c|}
\hline Táxon & Nome em português & REG & Localidade na EESG & UCs adjacentes \\
\hline Xenopsaris albinucha & Tijerila & - & - & APA \\
\hline \multicolumn{5}{|l|}{ Vireonidae } \\
\hline Cyclarhis gujanensis* & Pitiguari & $\mathrm{COL}$ & $1,2,3,5,6$ & APA, PEJ \\
\hline Vireo olivaceus & Juruviara & $\mathrm{AU}$ & $1,3,5,6$ & APA, PEJ \\
\hline Hylophilus amaurocephalus & Vite-vite-de-olho-cinza & - & - & APA \\
\hline Hylophilus pectoralis & Vite-vite-de-cabeça-cinza & - & - & PEJ \\
\hline \multicolumn{5}{|l|}{ Corvidae } \\
\hline Cyanocorax cristatellus* & Gralha-do-campo & $\mathrm{COL}$ & $1,3,5,6$ & APA, PEJ \\
\hline Cyanocorax cyanopogon & Gralha-cancã & $\mathrm{COL}$ & 1,2 & APA, PEJ \\
\hline \multicolumn{5}{|l|}{ Hirundinidae } \\
\hline Tachycineta leucorrhoa & Andorinha-de-sobre-branco & $\mathrm{O}$ & 3,6 & - \\
\hline Tachycineta albiventer & Andorinha-do-rio & - & - & APA, PEJ \\
\hline Progne tapera & Andorinha-do-campo & $\mathrm{O}$ & 3 & PEJ \\
\hline Progne chalybea & Andorinha-doméstica-grande & $\mathrm{O}$ & $1,3,6$ & APA, PEJ \\
\hline Pygochelidon cyanoleuca & Andorinha-pequena-de-casa & - & - & PEJ \\
\hline Stelgidopteryx ruficollis & Andorinha-serradora & $\mathrm{O}$ & 1,3 & APA, PEJ \\
\hline Hirundo rustica & Andorinha-de-bando & $\mathrm{O}$ & 3 & - \\
\hline \multicolumn{5}{|l|}{ Troglodytidae } \\
\hline Troglodytes musculus & Corruíra & COL & $1,2,3,5$ & APA, PEJ \\
\hline Pheugopedius genibarbis & Garrinchão-pai-avô & $\mathrm{AU}$ & $2,4,6$ & APA, PEJ \\
\hline Cantorchilus leucotis* & Garrinchão-de-barriga-vermelha & $\mathrm{COL}$ & $1,3,5,6$ & APA, PEJ \\
\hline \multicolumn{5}{|l|}{ Donacobiidae } \\
\hline Donacobius atricapillus & Japacanim & - & - & APA, PEJ \\
\hline \multicolumn{5}{|l|}{ Polioptilidae } \\
\hline Polioptila dumicola* & Balança-rabo-de-máscara & $\mathrm{COL}$ & $1,2,3,5,6$ & PEJ \\
\hline Polioptila plumbea & Balança-rabo-de-chapéu-preto & - & - & APA \\
\hline \multicolumn{5}{|l|}{ Turdidae } \\
\hline Turdus rufiventris & Sabiá-laranjeira & - & - & APA, PEJ \\
\hline Turdus leucomelas & Sabiá-barranco & COL & $1,2,3,5,6$ & APA, PEJ \\
\hline Turdus amaurochalinus & Sabiá-poca & $\mathrm{O}$ & 1 & APA, PEJ \\
\hline Turdus albicollis & Sabiá-coleira & - & - & PEJ \\
\hline \multicolumn{5}{|l|}{ Mimidae } \\
\hline Mimus saturninus* & Sabiá-do-campo & $\mathrm{COL}$ & $1,2,3,5,6$ & APA, PEJ \\
\hline \multicolumn{5}{|l|}{ Coerebidae } \\
\hline Coereba flaveola & Cambacica & $\mathrm{COL}$ & $1,3,5,6$ & APA, PEJ \\
\hline \multicolumn{5}{|l|}{ Thraupidae } \\
\hline Saltator maximus & Tempera-viola & $\mathrm{O}$ & 1 & APA, PEJ \\
\hline Saltator coerulescens & Sabiá-gongá & - & - & APA \\
\hline Saltator similis & Trinca-ferro-verdadeiro & - & - & PEJ \\
\hline Saltatricula atricollis* & Bico-de-pimenta & $\mathrm{COL}$ & $1,3,5$ & PEJ \\
\hline Schistochlamys ruficapillus & Bico-de-veludo & - & - & APA, PEJ \\
\hline Neothraupis fasciata & Cigarra-do-campo & $\mathrm{COL}$ & $1,2,3,5,6$ & APA, PEJ \\
\hline Compsothraupis loricata & Carretão & - & - & APA \\
\hline Thlypopsis sordida & Saí-canário & - & - & APA \\
\hline Cypsnagra hirundinacea & Bandoleta & $\mathrm{COL}$ & $1,2,3,5,6$ & APA, PEJ \\
\hline Tachyphonus rufus* & Pipira-preta & $\mathrm{O}$ & 1 & APA, PEJ \\
\hline Ramphocelus carbo* & Pipira-vermelha & $\mathrm{COL}$ & $1,2,3,5,6$ & APA, PEJ \\
\hline Thraupis sayaca & Sanhaçu-cinzento & $\mathrm{O}$ & $1,3,5,6$ & APA, PEJ \\
\hline Thraupis palmarum* & Sanhaçu-do-coqueiro & $\mathrm{COL}$ & $1,2,3,5,6$ & APA, PEJ \\
\hline Tangara cayana & Saíra-amarela & COL & $1,2,3,5,6$ & APA, PEJ \\
\hline Tersina viridis & Saí-andorinha & - & - & PEJ \\
\hline
\end{tabular}


Apêndice 1. Continuação...

\begin{tabular}{|c|c|c|c|c|}
\hline Táxon & Nome em português & REG & Localidade na EESG & UCs adjacentes \\
\hline Dacnis cayana & Saí-azul & $\mathrm{O}$ & $1,3,5,6$ & APA, PEJ \\
\hline Cyanerpes cyaneus & Saíra-beija-flor & $\mathrm{O}$ & 5 & - \\
\hline Hemithraupis guira & Saíra-de-papo-preto & $\mathrm{COL}$ & 1 & APA, PEJ \\
\hline Conirostrum speciosum & Figuinha-de-rabo-castanho & - & - & APA, PEJ \\
\hline Nemosia pileata & Saíra-de-chapéu-preto & - & - & APA, PEJ \\
\hline Eucometis penicillata & Pipira-da-taoca & - & - & PEJ \\
\hline \multicolumn{5}{|l|}{ Emberizidae } \\
\hline Zonotrichia capensis & Tico-tico & $\mathrm{COL}$ & $1,3,5,6$ & APA, PEJ \\
\hline Ammodramus humeralis* & Tico-tico-do-campo & $\mathrm{COL}$ & $1,2,3,5,6$ & APA, PEJ \\
\hline Ammodramus aurifrons & Cigarrinha-do-campo & - & - & PEJ \\
\hline Porphyrospiza caerulescens & Campainha-azul & - & - & APA, PEJ \\
\hline Sicalis citrina* & Canário-rasteiro & $\mathrm{O}$ & 1,5 & PEJ \\
\hline Sicalis columbiana & Canário-do-amazonas & $\mathrm{O}$ & 1 & APA \\
\hline Sicalis flaveola & Canário-da-terra-verdadeiro & - & - & APA \\
\hline Emberizoides herbicola* & Canário-do-campo & COL & $1,2,3,6$ & APA, PEJ \\
\hline Volatinia jacarina & Tiziu & $\mathrm{O}$ & $1,2,3,5,6$ & APA, PEJ \\
\hline Sporophila plumbea* & Patativa & $\mathrm{COL}$ & $1,2,3,5,6$ & APA, PEJ \\
\hline Sporophila lineola & Bigodinho & $\mathrm{COL}$ & 3 & APA \\
\hline Sporophila nigricollis & Baiano & $\mathrm{O}$ & 1,5 & APA, PEJ \\
\hline Sporophila leucoptera & Chorão & $\mathrm{COL}$ & 3 & - \\
\hline Sporophila bouvreuil & Caboclinho & $\mathrm{O}$ & 3 & - \\
\hline Sporophila castaneiventris & Caboclinho-de-peito-castanho & $\mathrm{O}$ & 1 & - \\
\hline Sporophila angolensis* & Curió & $\mathrm{COL}$ & $1,3,5$ & APA, PEJ \\
\hline Arremon taciturnus* & Tico-tico-de-bico-preto & $\mathrm{O}$ & 1,2 & APA, PEJ \\
\hline Charitospiza еисоsта* & Mineirinho & $\mathrm{COL}$ & $1,2,3,5$ & APA, PEJ \\
\hline Coryphospingus pileatus & Tico-tico-rei-cinza & $\mathrm{O}$ & 1 & APA \\
\hline Paroaria dominicana & Cardeal-do-nordeste & $\mathrm{O}$ & $2,4,6$ & - \\
\hline \multicolumn{5}{|l|}{ Cardinalidae } \\
\hline Piranga flava* & Sanhaçu-de-fogo & $\mathrm{COL}$ & 1 & APA, PEJ \\
\hline \multicolumn{5}{|l|}{ Parulidae } \\
\hline Parula pitiayumi & Mariquita & $\mathrm{O}$ & 3,6 & APA, PEJ \\
\hline Geothlypis aequinoctialis* & Pia-cobra & $\mathrm{O}$ & 1,3 & PEJ \\
\hline Basileuterus culicivorus & Pula-pula & $\mathrm{O}$ & 3,6 & APA \\
\hline Basileuterus flaveolus* & Canário-do-mato & COL & 1,2 & APA, PEJ \\
\hline Basileuterus hypoleucus & Pula-pula-de-barriga-branca & - & - & PEJ \\
\hline Basileuterus leucophrys* & Pula-pula-de-sobrancelha & $\mathrm{COL}$ & 1 & PEJ \\
\hline \multicolumn{5}{|l|}{ Icteridae } \\
\hline Psarocolius decumanus & Japu & $\mathrm{O}$ & 1,2 & APA, PEJ \\
\hline Cacicus cela & Xexéu & $\mathrm{O}$ & 1 & APA \\
\hline Icterus cayanensis* & Encontro & $\mathrm{O}$ & 1,5 & APA, PEJ \\
\hline Icterus jamacaii & Corrupião & - & - & APA \\
\hline Gnorimopsar chopi & Graúna & $\mathrm{COL}$ & $1,2,3,5,6$ & APA, PEJ \\
\hline Agelaioides fringillarius & Asa-de-telha-pálido & $\mathrm{O}$ & 3 & APA \\
\hline Molothrus bonariensis & Vira-bosta & - & - & APA, PEJ \\
\hline Molothrus rufoaxillaris & Vira-bosta-picumã & $\mathrm{O}$ & 3 & - \\
\hline Sturnella superciliaris & Polícia-inglesa-do-sul & $\mathrm{COL}$ & 3,7 & - \\
\hline \multicolumn{5}{|l|}{ Fringillidae } \\
\hline Sporagra magellanica & Pintassilgo & $\mathrm{COL}$ & 1,5 & APA, PEJ \\
\hline Euphonia chlorotica* & Fim-fim & $\mathrm{O}$ & $1,2,3,5$ & APA, PEJ \\
\hline \multicolumn{5}{|l|}{ Passeridae } \\
\hline Passer domesticus & Pardal & $\mathrm{O}$ & 1 & APA \\
\hline
\end{tabular}

